Research Article

\title{
Fuzzy Backstepping Sliding Mode Control for Mismatched Uncertain System
}

\author{
H. Q. Hou ${ }^{1, *}$, Q. Miao ${ }^{1}$, Q. H. Gao ${ }^{1}$ and Aorue, Bunam T. ${ }^{2}$ \\ ${ }^{1} \mathrm{Xi}$ 'an Research Inst. of hi-Tech hongqing Town, Xi'an, 710025, China \\ ${ }^{2}$ Intelligent Control and Data Analysis Center (IMAC) of BIMD Inc, Los Angeles, CA 90085-5429, United States
}

Received 1 March 2014; Accepted 25 June 2014

\begin{abstract}
Sliding mode controllers have succeeded in many control problems that the conventional control theories have difficulties to deal with; however it is practically impossible to achieve high-speed switching control. Therefore, in this paper an adaptive fuzzy backstepping sliding mode control scheme is derived for mismatched uncertain systems. Firstly fuzzy sliding mode controller is designed using backstepping method based on the Lyapunov function approach, which is capable of handling mismatched problem. Then fuzzy sliding mode controller is designed using T-S fuzzy model method, it can improve the performance of the control systems and their robustness. Finally this method of control is applied to nonlinear system as a case study; simulation results are also provided the performance of the proposed controller.
\end{abstract}

Keywords: Mismatched Uncertain System, T-S Fuzzy Model, Sliding Mode Control, Backstepping Control

\section{Introduction}

The problem of system control with uncertain has become a research focus. Sliding mode control has a wide application in system with matching uncertain, forever, its robustness is difficult to guarantee for mismatched system. An adaptive backstepping method is a systematic and recursive design methodology for nonlinear feedback control, there are unique structural design processes and capable of dealing with mismatched uncertainty, and successfully applied in aircraft, motor and machinery [1]. H. Bouadi designed sliding mode control based on backstepping approach, and applied in an UAV type-quadrotor[2].Literature[3] presented a backstepping SMC method to compensate the uncertainty which occurs in the slave system, and applied to implement synchronization of fractional-order Chaotic system. Farzin Piltan proposed a MIMO fuzzy backstepping adaptive fuzzy estimator variable structure controller design and application to engine in order to design high performance nonlinear controller [4]. In literature [5], Mohammad Reza Soltanpour presented controlling of a class of nonlinear systems with structured and unstructured uncertainties using fuzzy sliding mode control. Abdel Badie Sharkawy derived an adaptive fuzzy sliding mode control scheme for robotic systems [6]. However, the backstepping method requires the uncertainty of the systems can be parameterized representation, and with the relative degree increasing of controlled object, there is a calculated expansion problem, so that the controller is difficult to achieve.

The concept of Fuzzy control is to utilize the language rules of system to design a practical controller; it is

\footnotetext{
*E-mail address: 20016109@163.com
}

ISSN: 1791-2377 @ 2014 Kavala Institute of Technology. All rights reserved. particularly suitable for those systems with uncertain, timevarying and complex dynamics [7], [8]. T-S fuzzy model is a nonlinear model to describe the dynamic characteristics of complex systems, and more effective way to describe nonlinear systems [9]. For the T-S fuzzy model, Literature [10], [11], [12] also proved that there is better approximation performance than the Mamdani fuzzy model.

In this paper, an intelligent backstepping sliding mode control scheme is presented for MIMO nonlinear systems. The scheme is based on the universal approximation property of fuzzy systems and backstepping sliding mode control theory, and simulation was performed to validate the analysis result.

\section{Problem Statement}

This paper focuses on a class of MIMO nonlinear uncertain systems described as

$$
\left\{\begin{array}{l}
\dot{x}_{1}=b_{1} x_{2}+f_{1}\left(x_{1}\right) \\
\dot{x}_{2}=b_{2} u+f_{2}\left(x_{1}, x_{2}\right)+w(t)
\end{array}\right.
$$

Where $\boldsymbol{f}(\boldsymbol{x}) \in^{\circ}{ }^{n}$ is nonlinear function of the system, $\boldsymbol{x}=\left[\boldsymbol{x}_{1}^{\mathrm{T}}, \boldsymbol{x}_{2}^{\mathrm{T}}, \mathrm{L}, \boldsymbol{x}_{\mathrm{n}}^{\mathrm{T}}\right]^{\mathrm{T}}$ is the state variable of the system, $\boldsymbol{x}_{i}=\left[x_{i 1}, x_{i 2}, \mathrm{~L}, x_{i n}\right],(i=1,2, \mathrm{~L}, n) . \boldsymbol{u} \in^{\circ} n$ is the input vector of the system. $\boldsymbol{G}(\boldsymbol{x}) \neq 0$ and $\operatorname{rank}(\boldsymbol{x})=n ; \boldsymbol{w}(t)$ are the uncertainties and external disturbances, and it may not satisfy the so-called matching conditions. 
Assumption 1: There are constants of $\boldsymbol{b}_{j m}$ and $\boldsymbol{b}_{j M}$ to satisfy the inequality $0<\boldsymbol{b}_{j m}<\left|\boldsymbol{b}_{j}\right|<\boldsymbol{b}_{j M},(j=1,2)$, and converse matrix of matrix $\boldsymbol{b}_{j}$ exists.

The aim of the trajectory tracking control method is to design control law $\boldsymbol{u}(t)$, system state is eventually maintained at zero-from any initial state $\boldsymbol{x}(0) \neq 0$ in situations of uncertainty $\boldsymbol{w}(t)$.

\section{T-S Fuzzy Model}

T-S fuzzy model is a class of nonlinear model, and it is easy to describe the dynamic characteristics of complex systems.

Considering general nonlinear uncertain system, a compact T-S fuzzy model can be obtained by a careful selection of rule number and parameters [13], [14], [15]. The system is described as the following fuzzy rules, and then the fuzzy model is composed of the following rules

Plant Rules:

if $z_{1}$ is $F_{1}^{i}$ and $z_{2}$ is $F_{2}^{i} \mathrm{~L} z_{n}$ is $F_{n}^{i}$, then

$$
\begin{aligned}
& \dot{x}(t)=A_{i} x(t)+B_{i} u(t) \\
& y(t)=x(t)
\end{aligned}
$$

Where $\boldsymbol{z}(t)=\left[z_{1}(t), z_{2}(t), \mathrm{L}, z_{\mathrm{n}}(t)\right]^{T}$ is Fuzzy premise variables. $F_{i}^{l}$ is Fuzzy sets. $\boldsymbol{x}(t) \in \mathbf{R}^{n}$ is State variables. $\boldsymbol{u}(t) \in \mathbf{R}^{m}$ is the input of fuzzy system. $i=1,2, \mathrm{~L}, n$ is the number of system input. $\boldsymbol{y}(t) \in \mathbf{R}^{n}$ is Fuzzy output of the system. $\boldsymbol{A}_{i} \in \mathbf{R}^{n \times n}$ and $\boldsymbol{B}_{i} \in \mathbf{R}^{n \times m}$ are system vector and output vector.

Product inference engine, singleton fuzzifier and center average defuzzifier are used to calculate outputs. The fuzzy inferred output is written as

$$
\begin{aligned}
\dot{\boldsymbol{x}}(t) & =\sum_{i=1}^{n} \bar{a}_{i}(\boldsymbol{z}(t))\left[\boldsymbol{A}_{i} \boldsymbol{x}(t)+\boldsymbol{B}_{i} \boldsymbol{u}(t)\right] \\
\boldsymbol{y}(t)= & \sum_{i=1}^{n} \bar{a}_{i}(\boldsymbol{z}(t)) \boldsymbol{x}(t) \\
& \quad-\bar{\alpha}_{i}(\boldsymbol{z}(t))=\prod_{i=1}^{n} \mu_{F_{i}^{l}}(\boldsymbol{z}(t)) / \sum_{l=1}^{M} \prod_{i=1}^{n} \mu_{F_{i}^{\prime}}(\boldsymbol{z}(t)),
\end{aligned}
$$

$m_{F_{i}}$ is the membership function of $\boldsymbol{z}(t)$ in fuzzy set $F_{i}^{l}$.

Assuming the fuzzy system is controllable, then the fuzzy control rules is described as

$$
\begin{aligned}
& R^{i}: \text { if } z_{1} \text { is } F_{1}^{i} \text { and } z_{2} \text { is } F_{2}^{i} \mathrm{~L} z_{n} \text { is } F_{n}^{i}, \\
& \text { then } \boldsymbol{u}(t)=-\boldsymbol{K}_{i} \boldsymbol{x}(t)
\end{aligned}
$$
follows

The globally control law of state feedback is obtained as $\boldsymbol{u}(t)=\sum_{i=1}^{n} \bar{\alpha}_{i}(\boldsymbol{z}(t)) \boldsymbol{K}_{i} \boldsymbol{x}(t)$
Essentially, T-S fuzzy modelling method is approximation model, it is described by fuzzy IF-THEN rules in which the consequent parts represent local linear models.In order to get Fuzzy T-S model of nonlinear system, and it must obtain the linear model of each subsystem.

\section{Adaptive Backstepping Sliding Mode Control}

\subsection{Design of Tracking Controller}

The paper focuses on the formula (1) of the mismatched and uncertain system, this is a second-order system; it takes two steps to design adaptive backstepping sliding mode controller, the first step is to determine in the middle item with the stability $\tau_{1}$ by selecting a suitable Lyapunov function $\boldsymbol{V}$; the second step is to determine sliding mode control and parameter estimation algorithm according $V_{2}$.

\section{The first step:}

For the position tracking objective, define new state error vector $\boldsymbol{z}_{1}, \boldsymbol{z}_{2} \in R^{n}$ as

$$
\left\{\begin{array}{l}
z_{1}=x_{1}-x_{1 \mathrm{~d}} \\
z_{2}=x_{2}-\tau_{1}
\end{array}\right.
$$

Where $\boldsymbol{x}_{1 \mathrm{~d}}$ and $\tau_{1}$ are the desired state trajectory of the system, $\boldsymbol{x}_{1 \mathrm{~d}}$ is given by the control signal commands, and $\tau_{1}$ is a virtual control variable.

From (1) and (6), we can obtain as

$$
\left\{\begin{array}{l}
\dot{z}_{1}=b_{1} x_{2}+f_{1}\left(x_{1}\right)-\dot{x}_{1 \mathrm{~d}} \\
\dot{z}_{2}=b_{2} u+f_{2}\left(x_{1}, x_{2}\right)+w(t)-\dot{\tau}_{1}
\end{array}\right.
$$

Define virtual control variable $\tau_{1}$ as follows

$$
\tau_{1}=-b_{1}^{-1}\left(K_{1} z_{1}+f_{1}\left(x_{1}\right)-\dot{x}_{1 \mathrm{~d}}\right)
$$

Where $\boldsymbol{K}_{1}=\operatorname{diag}\left(k_{11}, k_{12}, \mathrm{~L}, k_{1 n}\right)$,

$k_{1 i}>0(i=1,2, \mathrm{~L}, n)$.

From (7) and (8), we can obtain

$$
\begin{aligned}
\dot{\boldsymbol{z}}_{1} & =\boldsymbol{b}_{1} \boldsymbol{x}_{2}+\boldsymbol{f}_{1}\left(\boldsymbol{x}_{1}\right)-\dot{\boldsymbol{x}}_{1 \mathrm{~d}} \\
& =-\boldsymbol{K}_{1} \boldsymbol{z}_{1}+\boldsymbol{b}_{1} \boldsymbol{z}_{2}
\end{aligned}
$$

Proposition 1: Using control law (8), the closed-loop system is global exponential stable based on assumption 1.

\section{Proof:}

Define the Lyapunov function as

$$
\boldsymbol{V}=\frac{1}{2} \boldsymbol{z}_{1}^{\mathrm{T}} \boldsymbol{z}_{1}+\frac{1}{2} \boldsymbol{z}_{2}^{\mathrm{T}} \boldsymbol{z}_{2} \text {, then we can obtain as follows }
$$

$$
\begin{aligned}
\dot{\boldsymbol{V}} & =\boldsymbol{z}_{1}^{\mathrm{T}} \dot{\boldsymbol{z}}_{1}+\boldsymbol{z}_{2}^{\mathrm{T}} \dot{\boldsymbol{z}}_{2} \\
& =\boldsymbol{z}_{1}^{\mathrm{T}}\left(-\boldsymbol{K}_{1} \boldsymbol{z}_{1}+\boldsymbol{b}_{1} \boldsymbol{z}_{2}\right)+\boldsymbol{z}_{2}^{\mathrm{T}}\left(\boldsymbol{b}_{2} \boldsymbol{u}\right. \\
& \left.+\boldsymbol{f}_{2}\left(\boldsymbol{x}_{1}, \boldsymbol{x}_{2}\right)+\boldsymbol{w}_{2}(t)-\dot{\boldsymbol{\tau}}_{1}\right)
\end{aligned}
$$


From assumption 1, converse matrix of matrix $\boldsymbol{b}_{j}$ exists, assuming upper bound of uncertainty and interference General items $\boldsymbol{w}(t)$ is $\overline{\boldsymbol{w}}$, and then the control law is

$$
\begin{aligned}
& \boldsymbol{u}=-\boldsymbol{b}_{2}^{-1}\left[\boldsymbol{K}_{2}\left(-\boldsymbol{K}_{1} \boldsymbol{z}_{1}+\boldsymbol{b}_{1} \boldsymbol{z}_{2}\right)+\boldsymbol{f}_{2}\left(\boldsymbol{x}_{1}, \boldsymbol{x}_{2}\right)\right. \\
& \left.+\overline{\boldsymbol{w}}-\dot{\boldsymbol{\tau}}_{1}+\boldsymbol{b}_{1}^{\mathrm{T}} \boldsymbol{z}_{1}\right]
\end{aligned}
$$

From control law (11) and (10), we obtain

$$
\dot{\boldsymbol{V}}=-\boldsymbol{z}_{1}^{\mathrm{T}} \boldsymbol{K}_{1} \boldsymbol{z}_{1}-\boldsymbol{z}_{2}^{\mathrm{T}} \boldsymbol{K}_{2} \boldsymbol{z}_{2}
$$

Through the Lyapunov stability theorem, thus the system is global exponential stable.

The second step:

Let $\boldsymbol{K}_{2}=\operatorname{diag}\left(k_{21}, k_{22}, \mathrm{~L}, k_{2 n}\right), k_{2 i}>0$.

Define the sliding surface function as follows

$$
s=K_{2} z_{1}+z_{2}
$$

Define Lyapunov function as $\boldsymbol{V}_{1}=\frac{1}{2} \boldsymbol{z}_{1}^{\mathrm{T}} \boldsymbol{z}_{1}+\frac{1}{2} \boldsymbol{s}^{\mathrm{T}} \boldsymbol{s}$, and then we can obtain its derivative as follows

$$
\begin{aligned}
\dot{V}_{1} & =\boldsymbol{z}_{1}^{\mathrm{T}} \dot{\boldsymbol{z}}_{1}+\boldsymbol{s}^{\mathrm{T}} \dot{\boldsymbol{s}} \\
& =\boldsymbol{z}_{1}^{\mathrm{T}}\left(-\boldsymbol{K}_{1} \boldsymbol{z}_{1}+\boldsymbol{b}_{1} \boldsymbol{z}_{2}\right)+\boldsymbol{s}^{\mathrm{T}}\left(\boldsymbol{K}_{2} \dot{\boldsymbol{z}}_{1}+\dot{\boldsymbol{z}}_{2}\right) \\
& =-\boldsymbol{z}_{1}^{\mathrm{T}} \boldsymbol{K}_{1} \boldsymbol{z}_{1}+\boldsymbol{z}_{1}^{\mathrm{T}} \boldsymbol{b}_{1} \boldsymbol{z}_{2}+\boldsymbol{s}^{\mathrm{T}}\left[\boldsymbol { K } _ { 2 } \left(-\boldsymbol{K}_{1} \boldsymbol{z}_{1}\right.\right. \\
& \left.\left.+\boldsymbol{b}_{1} \boldsymbol{z}_{2}\right)+\boldsymbol{b}_{2} \boldsymbol{u}+\boldsymbol{f}_{2}\left(\boldsymbol{x}_{1}, \boldsymbol{x}_{2}\right)+\boldsymbol{w}_{2}(t)-\dot{\boldsymbol{\tau}}_{1}\right]
\end{aligned}
$$

From assumption 1, converse matrix of matrix $\boldsymbol{b}_{j}$ exists, assuming upper bound of uncertainty and interference General items $\boldsymbol{w}(t)$ is $\overline{\boldsymbol{w}}$, and then the control law is

$$
\begin{aligned}
& \boldsymbol{u}=-\boldsymbol{b}_{2}^{-1}\left[\boldsymbol{K}_{2}\left(-\boldsymbol{K}_{1} z_{1}+\boldsymbol{b}_{1} \boldsymbol{z}_{2}\right)+\boldsymbol{f}_{2}\left(\boldsymbol{x}_{1}, \boldsymbol{x}_{2}\right)\right. \\
& \left.+\bar{w}-\dot{\tau}_{1}+\boldsymbol{K}_{3} s+\boldsymbol{K}_{4} \operatorname{sgn}(s)\right]
\end{aligned}
$$

Assuming upper bound of uncertainty and interference General items is $\boldsymbol{w}(t)$, it is unknown and easy to cause chattering problem. In order to avoid using its upper bound, and we should use adaptive algorithm to estimate $\boldsymbol{w}(t)$, then we can design an estimates $\dot{\hat{\boldsymbol{w}}}$ as follows

$$
\dot{\hat{\boldsymbol{w}}}=\boldsymbol{K}_{5} \boldsymbol{s}^{\mathrm{T}}
$$

We obtain adaptive control law as follows

$$
\begin{aligned}
& u=-b_{2}^{-1}\left[K_{2}\left(-K_{1} z_{1}+b_{1} z_{2}\right)+f_{2}\left(x_{1}, x_{2}\right)\right. \\
& \left.+\hat{w}-\dot{\tau}_{1}+K_{3} s+K_{4} \operatorname{sgn}(s)\right]
\end{aligned}
$$

$$
\begin{aligned}
& \quad \text { Where } \boldsymbol{K}_{3}=\operatorname{diag}\left(k_{31}, k_{32}, \mathrm{~L}, k_{3 n}\right), \\
& \boldsymbol{K}_{4}=\operatorname{diag}\left(k_{41}, k_{42}, \mathrm{~L}, k_{4 n}\right), \\
& \boldsymbol{K}_{5}=\operatorname{diag}\left(k_{51}, k_{52}, \mathrm{~L}, k_{5 n}\right) \\
& k_{j i}>0(i=1,2, \mathrm{~L}, n, j=3,4,5) .
\end{aligned}
$$

\subsection{Stability Analysis}

Define Lyapunov function as follows

$\boldsymbol{V}=\frac{1}{2} \boldsymbol{z}_{1}^{\mathrm{T}} \boldsymbol{z}_{1}+\frac{1}{2} \boldsymbol{s}^{\mathrm{T}} \boldsymbol{s}+\frac{1}{2 \boldsymbol{K}_{5}} \tilde{\boldsymbol{w}}^{2}$

Assuming uncertainties $\boldsymbol{w}(t)$, its estimated value is $\hat{\boldsymbol{w}}$, and its estimated error is $\boldsymbol{W} \sigma \boldsymbol{w}^{*}-\hat{\boldsymbol{w}}, \boldsymbol{K}_{5}$ is a positive definite diagonal matrix. The derivative of Lyapunov function is written as

$$
\begin{aligned}
\dot{V}_{1}= & \boldsymbol{z}_{1}^{\mathrm{T}} \dot{\boldsymbol{z}}_{1}+\boldsymbol{s}^{\mathrm{T}} \dot{\boldsymbol{s}}+\tilde{\boldsymbol{w}}^{2} / 2 \boldsymbol{K}_{5} \\
& =\boldsymbol{z}_{1}^{\mathrm{T}}\left(-\boldsymbol{K}_{1} \boldsymbol{z}_{1}+\boldsymbol{b}_{1} \boldsymbol{z}_{2}\right)+\boldsymbol{s}^{\mathrm{T}}\left(\boldsymbol{K}_{2} \dot{\boldsymbol{z}}_{1}+\dot{\boldsymbol{z}}_{2}\right)-\tilde{\boldsymbol{w}} \dot{\hat{\boldsymbol{w}}} / \boldsymbol{K}_{5} \\
& =-\boldsymbol{z}_{1}^{\mathrm{T}} \boldsymbol{K}_{1} \boldsymbol{z}_{1}+\boldsymbol{z}_{1}^{\mathrm{T}} \boldsymbol{b}_{1} \boldsymbol{z}_{2}+\boldsymbol{s}^{\mathrm{T}}\left[\boldsymbol{K}_{2}\left(-\boldsymbol{K}_{1} \boldsymbol{z}_{1}+\boldsymbol{b}_{1} \boldsymbol{z}_{2}\right)\right. \\
& \left.+\boldsymbol{b}_{2} \boldsymbol{u}+\boldsymbol{f}_{2}\left(\boldsymbol{x}_{1}, \boldsymbol{x}_{2}\right)+\boldsymbol{w}_{2}(t)-\dot{\tau}_{1}\right]-\tilde{\boldsymbol{w}} \dot{\hat{\boldsymbol{w}}} / \boldsymbol{K}_{5} \\
& =-\boldsymbol{z}_{1}^{\mathrm{T}} \boldsymbol{K}_{1} \boldsymbol{z}_{1}+\boldsymbol{z}_{1}^{\mathrm{T}} \boldsymbol{b}_{1} \boldsymbol{z}_{2}+\boldsymbol{s}^{\mathrm{T}}\left[\boldsymbol{K}_{2}\left(-\boldsymbol{K}_{1} \boldsymbol{z}_{1}+\boldsymbol{b}_{1} \boldsymbol{z}_{2}\right)\right. \\
& \left.+\boldsymbol{b}_{2} \boldsymbol{u}+\boldsymbol{f}_{2}\left(\boldsymbol{x}_{1}, \boldsymbol{x}_{2}\right)+\hat{\boldsymbol{w}}-\dot{\tau}_{1}\right]-\tilde{\boldsymbol{w}}\left(\dot{\hat{\boldsymbol{w}}}-\boldsymbol{K}_{5} \boldsymbol{s}^{\mathrm{T}}\right) / \boldsymbol{K}_{5}
\end{aligned}
$$

From control law formula (17), adaptive law formula (16) and (19), we obtain

$$
\begin{gathered}
\dot{\boldsymbol{V}}_{1}=-\boldsymbol{z}_{1}^{\mathrm{T}} \boldsymbol{K}_{1} \boldsymbol{z}_{1}+\boldsymbol{z}_{1}^{\mathrm{T}} \boldsymbol{b}_{1} \boldsymbol{z}_{2}-\boldsymbol{s}^{\mathrm{T}} \boldsymbol{K}_{3} \boldsymbol{s}-\boldsymbol{K}_{4}|\boldsymbol{s}| \\
\text { Selecting } \boldsymbol{Q}=\left(\begin{array}{cc}
\boldsymbol{K}_{1}+\boldsymbol{K}_{2} \boldsymbol{K}_{3} \boldsymbol{K}_{2} & \boldsymbol{K}_{2} \boldsymbol{K}_{3}+\boldsymbol{b}_{1} \\
\boldsymbol{K}_{3} \boldsymbol{K}_{2} & \boldsymbol{K}_{3}
\end{array}\right), \text { and }
\end{gathered}
$$

let $\boldsymbol{z}^{\mathrm{T}}=\left[\begin{array}{ll}\boldsymbol{z}_{1}^{\mathrm{T}} & \boldsymbol{z}_{2}^{\mathrm{T}}\end{array}\right], \boldsymbol{z}=\left[\begin{array}{l}\boldsymbol{z}_{1} \\ \boldsymbol{z}_{2}\end{array}\right]$, then

$$
\begin{aligned}
\boldsymbol{z}^{\mathrm{T}} \boldsymbol{Q} \boldsymbol{z} & =\left[\begin{array}{ll}
\boldsymbol{z}_{1}^{\mathrm{T}} & \boldsymbol{z}_{2}^{\mathrm{T}}
\end{array}\right]\left(\begin{array}{cc}
\boldsymbol{K}_{1}+\boldsymbol{K}_{2} \boldsymbol{K}_{3} \boldsymbol{K}_{2} & \boldsymbol{K}_{2} \boldsymbol{K}_{3}+\boldsymbol{b}_{1} \\
\boldsymbol{K}_{3} \boldsymbol{K}_{2} & \boldsymbol{K}_{3}
\end{array}\right)\left[\begin{array}{l}
\boldsymbol{z}_{1} \\
\boldsymbol{z}_{2}
\end{array}\right] \\
= & \boldsymbol{z}_{1}^{\mathrm{T}} \boldsymbol{K}_{1} \boldsymbol{z}_{1}-\boldsymbol{z}_{1}^{\mathrm{T}} \boldsymbol{b}_{1} \boldsymbol{z}_{2}+\boldsymbol{z}_{1}^{\mathrm{T}} \boldsymbol{K}_{2} \boldsymbol{K}_{3} \boldsymbol{K}_{2} \boldsymbol{z}_{1}+\boldsymbol{z}_{1}^{\mathrm{T}} \boldsymbol{K}_{2} \boldsymbol{K}_{3} \boldsymbol{z}_{2} \\
& +\boldsymbol{z}_{2}^{\mathrm{T}} \boldsymbol{K}_{3} \boldsymbol{K}_{2} \boldsymbol{z}_{1}+\boldsymbol{z}_{2}^{\mathrm{T}} \boldsymbol{K}_{3} \boldsymbol{z}_{2}
\end{aligned}
$$

From (20), we obtain

$$
\begin{aligned}
\dot{V}_{1} & =-\boldsymbol{z}_{1}^{\mathrm{T}} \boldsymbol{K}_{1} \boldsymbol{z}_{1}+\boldsymbol{z}_{1}^{\mathrm{T}} \boldsymbol{b}_{1} \boldsymbol{z}_{2}-\boldsymbol{s}^{\mathrm{T}} \boldsymbol{K}_{3} \boldsymbol{s}-\boldsymbol{K}_{4}|\boldsymbol{s}| \\
& =-\boldsymbol{z}^{\mathrm{T}} \boldsymbol{Q}_{\boldsymbol{z}}-\boldsymbol{K}_{4}|\boldsymbol{s}|
\end{aligned}
$$

By choosing the appropriate parameter value, we can obtain $|\boldsymbol{Q}|>0$, namely $\boldsymbol{Q}$ is a positive definite matrix, which can make $\boldsymbol{I}_{1}^{\&} \leq 0$. Thus, we can guarantee that control system is stable.

\section{Backstepping Sliding Mode Control Based on T-S fuzzy model}

\subsection{Fuzzy Backstepping Sliding Mode controller design} Based on the approximation property of T-S fuzzy systems, a nonlinear uncertain system can be expressed as the form of linear parametric model and a modeling error term; the 
system makes full use of the advantages of fuzzy control and linear control.

The paper focuses on the formula (1) of uncertain system, which is described based on T-S fuzzy dynamical model. We obtain $r$ fuzzy rules, and then the fuzzy model is composed of the following rules

\section{Plant Rules $i$ :}

if $z_{1}$ is $F_{1}^{i}$ and $z_{2}$ is $F_{2}^{i} \cdots z_{n}$ is $F_{n}^{i}$, then

$$
\left\{\begin{array}{l}
\dot{\boldsymbol{x}}_{1}=\boldsymbol{b}_{1 i} \boldsymbol{x}_{2}+\boldsymbol{f}_{1 i}\left(\boldsymbol{x}_{1}\right) \\
\dot{\boldsymbol{x}}_{2}=\boldsymbol{b}_{2 i} \boldsymbol{u}+\boldsymbol{f}_{2 i}\left(\boldsymbol{x}_{1}, \boldsymbol{x}_{2}\right)+\boldsymbol{w}_{i}(t)
\end{array}\right.
$$

Where $\boldsymbol{z}(t)=\left[z_{1}(t), z_{2}(t), \mathrm{L}, z_{\mathrm{n}}(t)\right]^{T}$ is Fuzzy premise variable. $F_{i}^{l}$ is Fuzzy sets. $\boldsymbol{x}(t) \in \mathbf{R}^{n}$ is State variable. $\boldsymbol{u}(t) \in \mathbf{R}^{m}$ is the input of fuzzy system. $\boldsymbol{f}_{j i}$ and $\boldsymbol{b}_{j i}$ are nonlinear function matrix. $\boldsymbol{w}(t)$ are the uncertainties and external disturbances.

Assuming that $\alpha_{i}\left(t_{i}\right)$ is membership function of $z_{i}$ in fuzzy set $F^{i}$, then the global T-S fuzzy model of nonlinear uncertain system is written as

$$
\left\{\begin{array}{l}
\dot{\boldsymbol{x}}_{1}=\sum_{i=1}^{r}-\bar{\alpha}_{i}\left(z_{i}\right)\left(\boldsymbol{b}_{1} \boldsymbol{x}_{2}+\boldsymbol{f}_{1}\left(\boldsymbol{x}_{1}\right)\right) \\
\dot{\boldsymbol{x}}_{2}=\sum_{i=1}^{r}-\bar{\alpha}_{i}\left(z_{i}\right)\left(\boldsymbol{b}_{2} \boldsymbol{u}+\boldsymbol{f}_{2}\left(\boldsymbol{x}_{1}, \boldsymbol{x}_{2}\right)+\boldsymbol{w}(t)\right) \\
\text { Where } \overline{\boldsymbol{\alpha}}_{i}(\boldsymbol{z}(t))=\alpha_{i}(\boldsymbol{z}(t)) / \sum_{i=1}^{r} \alpha_{i}(\boldsymbol{z}(t)) .
\end{array}\right.
$$

Design step of controller Based on Backstepping Sliding Mode method is written as

\section{The first step:}

Define new error state vector as $\boldsymbol{z}_{1}, \boldsymbol{z}_{2} \in R^{n}$, then there are the following formula

$$
\left\{\begin{array}{l}
z_{1}=x_{1}-x_{1 d} \\
z_{2}=x_{2}-\tau_{1}
\end{array}\right.
$$

Where $\boldsymbol{x}_{1 \mathrm{~d}}$ and $\tau_{1}$ are the desired state trajectory of the system, and $\boldsymbol{x}_{1 \mathrm{~d}}$ is the input signal of the system.

Assuming that $\tau_{1}$ is virtual control variable, From Equation (24) and Equation (25), we can obtain

$$
\left\{\begin{array}{l}
\dot{\boldsymbol{z}}_{1}=\boldsymbol{b}_{1 i} \boldsymbol{x}_{2}+\boldsymbol{f}_{1 i}\left(\boldsymbol{x}_{1}\right)-\dot{\boldsymbol{x}}_{1 \mathrm{~d}} \\
\dot{\boldsymbol{z}}_{2}=\boldsymbol{b}_{2 i} \boldsymbol{u}+\boldsymbol{f}_{2 i}\left(\boldsymbol{x}_{1}, \boldsymbol{x}_{2}\right)+\boldsymbol{w}_{i}(t)-\dot{\boldsymbol{\tau}}_{1}
\end{array}\right.
$$

Selecting virtual control variable $\tau_{1}$ as follows

$$
\tau_{1}=-b_{1 i}^{-1}\left(K_{1} z_{1}+f_{1 i}\left(x_{1}\right)-\dot{x}_{1 \mathrm{~d}}\right)
$$

Where $\boldsymbol{K}_{1}=\operatorname{diag}\left(k_{11}, k_{12}, \mathrm{~L}, k_{1 n}\right)$,

$k_{1 i}>0(i=1,2, \mathrm{~L}, n)$.

From (26) and (27), we obtain

$$
\begin{aligned}
\dot{\boldsymbol{z}}_{1} & =\boldsymbol{b}_{1 i} \boldsymbol{x}_{2}+\boldsymbol{f}_{1 i}\left(\boldsymbol{x}_{1}\right)-\dot{\boldsymbol{x}}_{1 \mathrm{~d}} \\
& =-\boldsymbol{K}_{1} \boldsymbol{z}_{1}+\boldsymbol{b}_{1 i} \boldsymbol{z}_{2}
\end{aligned}
$$

Let $\boldsymbol{K}_{2}=\operatorname{diag}\left(k_{21}, k_{22}, \mathrm{~L}, k_{2 n}\right), k_{1 i}>0(i=1,2, \mathrm{~L}, n)$, and define the sliding surface function as follows

$s=K_{2} z_{1}+z_{2}$

Define Lyapunov function as $V_{1}=\frac{1}{2} z_{1}^{\mathrm{T}} \boldsymbol{z}_{1}+\frac{1}{2} \boldsymbol{s}^{\mathrm{T}} \boldsymbol{s}$, then we can obtain as follows

$$
\begin{aligned}
\dot{V}_{1}= & \boldsymbol{z}_{1}^{\mathrm{T}} \dot{\boldsymbol{z}}_{1}+\boldsymbol{s}^{\mathrm{T}} \dot{\boldsymbol{s}} \\
& =\boldsymbol{z}_{1}^{\mathrm{T}}\left(-\boldsymbol{K}_{1} \boldsymbol{z}_{1}+\boldsymbol{b}_{1 i} \boldsymbol{z}_{2}\right)+\boldsymbol{s}^{\mathrm{T}}\left(\boldsymbol{K}_{2} \dot{\boldsymbol{z}}_{1}+\dot{\boldsymbol{z}}_{2}\right) \\
& =-\boldsymbol{z}_{1}^{\mathrm{T}} \boldsymbol{K}_{1} \boldsymbol{z}_{1}+\boldsymbol{z}_{1}^{\mathrm{T}} \boldsymbol{b}_{1 i} \boldsymbol{z}_{2}+\boldsymbol{s}^{\mathrm{T}}\left[\boldsymbol { K } _ { 2 } \left(-\boldsymbol{K}_{1} \boldsymbol{z}_{1}\right.\right. \\
& \left.\left.+\boldsymbol{b}_{1 i} \boldsymbol{z}_{2}\right)+\boldsymbol{b}_{2 i} \boldsymbol{u}+\boldsymbol{f}_{2 i}\left(\boldsymbol{x}_{1}, \boldsymbol{x}_{2}\right)+\boldsymbol{w}_{i}(t)-\dot{\boldsymbol{\tau}}_{1}\right]
\end{aligned}
$$

From (30), we obtain the backstepping sliding mode control Law based on T-S fuzzy model as follows

$$
\begin{aligned}
& \text { if } z_{1} \text { is } F_{1}^{i} \text { and } z_{2} \text { is } F_{2}^{i} \cdots z_{n} \text { is } F_{n}^{i} \text {, then } \\
& \begin{aligned}
\boldsymbol{u}_{i}= & -\boldsymbol{b}_{2 i}^{-1}\left[\boldsymbol{K}_{2}\left(-\boldsymbol{K}_{1} \boldsymbol{z}_{1}+\boldsymbol{b}_{1 i} \boldsymbol{z}_{2}\right)+\boldsymbol{f}_{2 i}\left(\boldsymbol{x}_{1}, \boldsymbol{x}_{2}\right)\right. \\
& \left.+\hat{\boldsymbol{w}}-\dot{\boldsymbol{\tau}}_{1 i}+\boldsymbol{K}_{3} \boldsymbol{s}+\boldsymbol{K}_{4} \operatorname{sgn}(\boldsymbol{s})\right]
\end{aligned}
\end{aligned}
$$

Then the adaptive law $\underset{\boldsymbol{w}}{\boldsymbol{\alpha}}$ is

$$
\stackrel{\&}{\mathscr{W}}=\boldsymbol{K}_{5} \boldsymbol{s}^{\mathrm{T}}
$$

Thus, the global control law is weighted sum of control law of local subsystem; we obtain the global control law as

$\boldsymbol{u}=\sum_{i=1}^{r} \bar{\alpha}_{i} \boldsymbol{u}_{i}$

\subsection{Stability Analysis}

Define the Lyapunov function as

$\boldsymbol{V}=\frac{1}{2} \boldsymbol{z}_{1}^{\mathrm{T}} \boldsymbol{z}_{1}+\frac{1}{2} \boldsymbol{s}^{\mathrm{T}} \boldsymbol{s}+\frac{1}{2 \boldsymbol{K}_{5}} \boldsymbol{w}^{\mathrm{T}} \mathrm{o}$, and the derivative of Lyapunov function is written as

$$
\begin{aligned}
\dot{\boldsymbol{V}}_{1} & =\boldsymbol{z}_{1}^{\mathrm{T}} \dot{\boldsymbol{z}}_{1}+\boldsymbol{s}^{\mathrm{T}} \dot{\boldsymbol{s}}+\frac{1}{2 \boldsymbol{K}_{5}} \tilde{\boldsymbol{w}}^{2} \\
& =-\boldsymbol{z}_{1}^{\mathrm{T}} \boldsymbol{K}_{1} \boldsymbol{z}_{1}+\boldsymbol{z}_{1}^{\mathrm{T}} \boldsymbol{b}_{1 i} \boldsymbol{z}_{2}+\boldsymbol{s}^{\mathrm{T}}\left[\boldsymbol{K}_{2}\left(-\boldsymbol{K}_{1} \boldsymbol{z}_{1}+\boldsymbol{b}_{1 i} \boldsymbol{z}_{2}\right)\right. \\
& \left.+\boldsymbol{b}_{2 i} \boldsymbol{u}+\boldsymbol{f}_{2 i}\left(\boldsymbol{x}_{1}, \boldsymbol{x}_{2}\right)+\hat{\boldsymbol{w}}-\dot{\boldsymbol{\tau}}_{1}\right]-\frac{1}{\boldsymbol{K}_{5}} \tilde{\boldsymbol{w}}\left(\dot{\hat{\boldsymbol{w}}}-\boldsymbol{K}_{5} \boldsymbol{s}^{\mathrm{T}}\right)
\end{aligned}
$$

From control law formula (31) and adaptive law formula (32) and (34), we obtain 


$$
\dot{\boldsymbol{V}}_{1}=-\boldsymbol{z}_{1}^{\mathrm{T}} \boldsymbol{K}_{1} \boldsymbol{z}_{1}+\boldsymbol{z}_{1}^{\mathrm{T}} \boldsymbol{b}_{1 i} \boldsymbol{z}_{2}-\boldsymbol{s}^{\mathrm{T}} \boldsymbol{K}_{3} \boldsymbol{s}-\boldsymbol{K}_{4}|\boldsymbol{s}|
$$

Then (35) can be rewritten as

$$
\begin{aligned}
\dot{V}_{1} & =-\boldsymbol{z}_{1}^{\mathrm{T}} \boldsymbol{K}_{1} \boldsymbol{z}_{1}+\boldsymbol{z}_{1}^{\mathrm{T}} \boldsymbol{b}_{1 i} \boldsymbol{z}_{2}-\boldsymbol{s}^{\mathrm{T}} \boldsymbol{K}_{3} \boldsymbol{s}-\boldsymbol{K}_{4}|\boldsymbol{s}| \\
& =-\boldsymbol{z}^{\mathrm{T}} \boldsymbol{Q} \boldsymbol{z}-\boldsymbol{K}_{4}|\boldsymbol{s}|
\end{aligned}
$$

By choosing the appropriate parameter value, we can obtain $\dot{\boldsymbol{V}}_{1} \leq 0$, and then we can guarantee that control subsystem is asymptotical stable.

Define Lyapunov function as follows

$$
\begin{aligned}
\dot{\boldsymbol{V}} & =\boldsymbol{z}_{1}^{\mathrm{T}} \dot{\boldsymbol{z}}_{1}+\boldsymbol{s}^{\mathrm{T}} \dot{\boldsymbol{s}}+\tilde{\boldsymbol{w}}^{2} / 2 \boldsymbol{K}_{5} \\
& =\sum_{i=1}^{r} \alpha_{i}\left[-\boldsymbol{z}_{1}^{\mathrm{T}} \boldsymbol{K}_{1} \boldsymbol{z}_{1}+\boldsymbol{z}_{1}^{\mathrm{T}} \boldsymbol{b}_{1 i} \boldsymbol{z}_{2}+\boldsymbol{s}^{\mathrm{T}}\left[\boldsymbol { K } _ { 2 } \left(-\boldsymbol{K}_{1} \boldsymbol{z}_{1}\right.\right.\right. \\
& \left.\left.+\boldsymbol{b}_{1 i} \boldsymbol{z}_{2}\right)+\boldsymbol{b}_{2 i} \boldsymbol{u}+\boldsymbol{f}_{2 i}\left(\boldsymbol{x}_{1}, \boldsymbol{x}_{2}\right)+\hat{\boldsymbol{w}}-\dot{\boldsymbol{\tau}}_{1}\right] \\
& \left.-\tilde{\boldsymbol{w}}\left(\dot{\hat{\boldsymbol{w}}}-\boldsymbol{K}_{5} \boldsymbol{s}^{\mathrm{T}}\right) / \boldsymbol{K}_{5}\right] \\
& =-\boldsymbol{z}^{\mathrm{T}} \boldsymbol{Q} \boldsymbol{z}-\boldsymbol{K}_{4}|\boldsymbol{s}|<0
\end{aligned}
$$

Thus, the global control system is asymptotically stable; then the asymptotical stability of system was proved based on Lyapunov theory.

\section{Simulation}

In this section, illustrative numerical simulation example is provided to demonstrate the effectiveness and robustness of the proposed approach. The system is represented as

$$
\ddot{q}=f(x)+b(x) u
$$

$$
\text { Where } \boldsymbol{f}(\boldsymbol{x})=\left[\begin{array}{l}
\frac{2 g \cos \left(q_{1}+q_{2}\right)\left(\sin q_{2}-1\right)}{\cos \left(2 q_{2}\right)+4 \cos q_{2}+19} \\
\frac{2 g \cos \left(q_{1}+q_{2}\right)\left(2 \cos q_{2}+10\right)}{\cos \left(2 q_{2}\right)+4 \cos q_{2}+19}
\end{array}\right] \text {, }
$$

$$
\boldsymbol{b}(\boldsymbol{x})=\left[\begin{array}{cc}
\frac{200}{\cos \left(2 q_{2}\right)+4 \cos q_{2}+19} & \frac{-200 \sin q_{2}}{\cos \left(2 q_{2}\right)+4 \cos q_{2}+19} \\
\frac{-200 \sin q_{2}}{\cos \left(2 q_{2}\right)+4 \cos q_{2}+19} & \frac{400 \cos q_{2}+2000}{\cos \left(2 q_{2}\right)+4 \cos q_{2}+19}
\end{array}\right] .
$$

In this paper, to verify the accuracy and usefulness of the method, it transforms the formula (38) to state space model, which it can be designed based on fuzzy backstepping sliding mode control method.

Let $\boldsymbol{x}_{1}=\left[q_{1}, q_{2}\right]^{\mathrm{T}}, \boldsymbol{x}_{2}=\left[\dot{q}_{1}, \dot{q}_{2}\right]^{\mathrm{T}}$, the state variable is $\boldsymbol{x}=\left[\boldsymbol{x}_{1}^{\mathrm{T}}, \boldsymbol{x}_{2}^{\mathrm{T}}\right]^{\mathrm{T}}=\left[q_{1}, q_{2}, \dot{q}_{1}, \dot{q}_{2}\right]^{\mathrm{T}}$.

From (38) can be written as follows

$$
\left\{\begin{array}{l}
\dot{x}_{1}=x_{2} \\
\dot{x}_{2}=f(x)+b(x) u+w(t)
\end{array}\right.
$$

Where $\boldsymbol{w}(t)$ are the uncertainties and external disturbances.
The following T-S fuzzy model is used to model of the nonlinear system.

\section{Rules:}

If $q_{1}$ is $-\pi / 2, \pm \pi / 2, \pi / 2,0,0, \pi / 2, \pi / 2$; and $q_{2}$ is $-\pi / 2,0,-\pi / 2, \pm \pi / 2,0,-\pi / 2,-\pi / 2$. (Here only give equation and parameter values at $q_{1}$ is $-\pi / 2$ and $q_{2}$ is $-\pi / 2$ ), then

$$
\left\{\begin{array}{l}
\dot{x}_{1}=x_{2} \\
\dot{x}_{2}=f_{i}(x)+b_{i}(x) u
\end{array}\right.
$$

$$
\begin{gathered}
\text { Where } \boldsymbol{f}_{1}=\left[\begin{array}{c}
1.0778-0.6861 q_{2} \\
-10.9637
\end{array}\right], \\
\boldsymbol{b}_{1}=\left[\begin{array}{cc}
11.0241 & -7.0182 q_{2} \\
-7.0182 q_{2} & 111.0109
\end{array}\right] ; \text { Other value of } \boldsymbol{f}_{i}
\end{gathered}
$$$$
\text { and } \boldsymbol{b}_{i} \text { are omitted, } i=1, \mathrm{~L} 7 \text {. }
$$

Assuming Command signal $q_{1 d}$ and $q_{2 d}$ of system are $q_{1 d}=\sin (0.4 \pi t)$ and $q_{2 d}=\sin (0.6 \pi t)$; the initial state of the system is $\boldsymbol{x}=[0.5,0.5,0,0]$.

The proposed control law is applied to controller design of system (38), control law using the article achieve the control of system, and defining parameters of control system are as follows

$$
\begin{aligned}
& K_{1}=\left[\begin{array}{cc}
10 & 0 \\
0 & 10
\end{array}\right], K_{2}=\left[\begin{array}{cc}
10 & 0 \\
0 & 10
\end{array}\right], \\
& K_{3}=\left[\begin{array}{ll}
1 & 0 \\
0 & 1
\end{array}\right], K_{4}=\left[\begin{array}{cc}
0.1 & 0 \\
0 & 0.1
\end{array}\right] .
\end{aligned}
$$

In this paper, the membership function of fuzzy logic control is Triangular membership function, which completes the fuzziness of input signal.

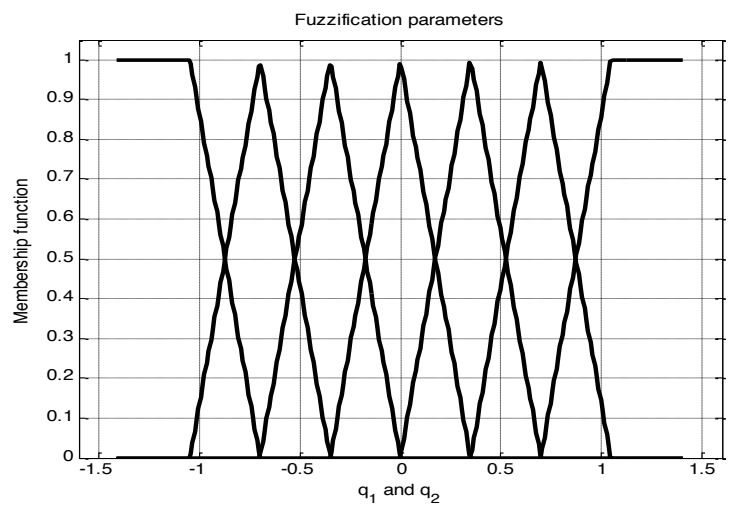

Fig. 1. Fuzzy membership function

At $\boldsymbol{w}(t)=0$, the results of simulation are shown in Figure 2 and Figure 3. 


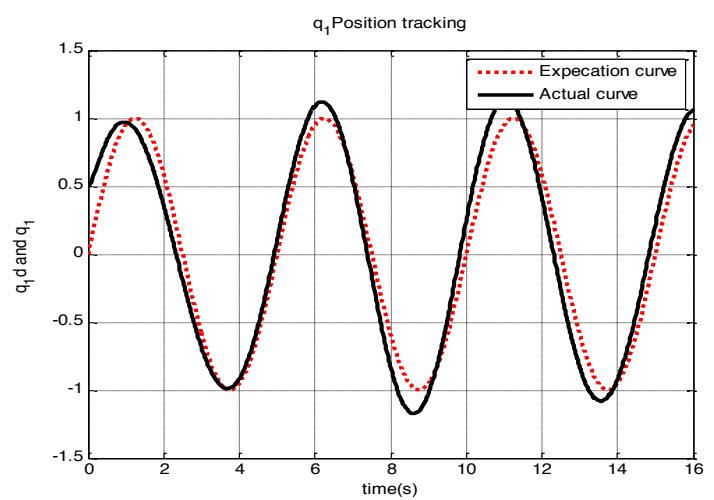

Fig. 2. Position tracking curve of $q_{1}$

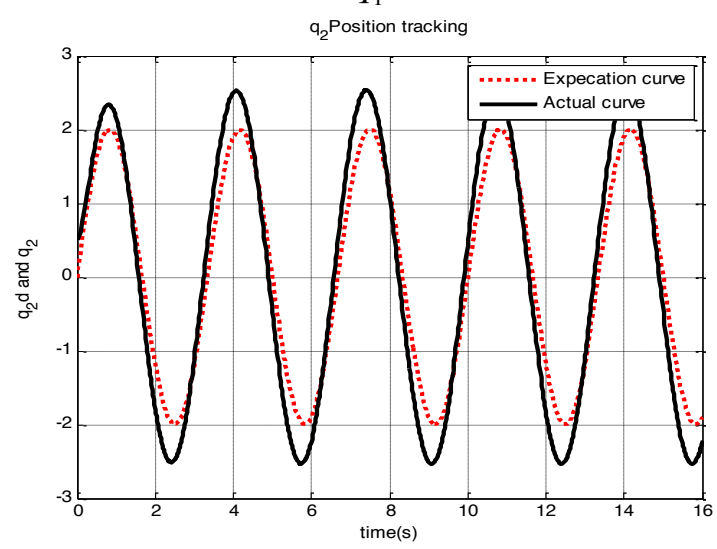

Fig. 3. Position tracking curve of $q_{2}$

$$
\text { At } \boldsymbol{w}(t)=\left[\begin{array}{c}
2 \sin \dot{q}_{1}+0.2 \dot{q}_{1} \\
2 \sin \dot{q}_{2}+0.2 \dot{q}_{2}
\end{array}\right] \text {, and we don't change }
$$

the value of the control parameters. The results of simulation are shown in Figures 4 and 5, In the Figure, the dotted line is the expectation curve and the solid line is the actual curve.

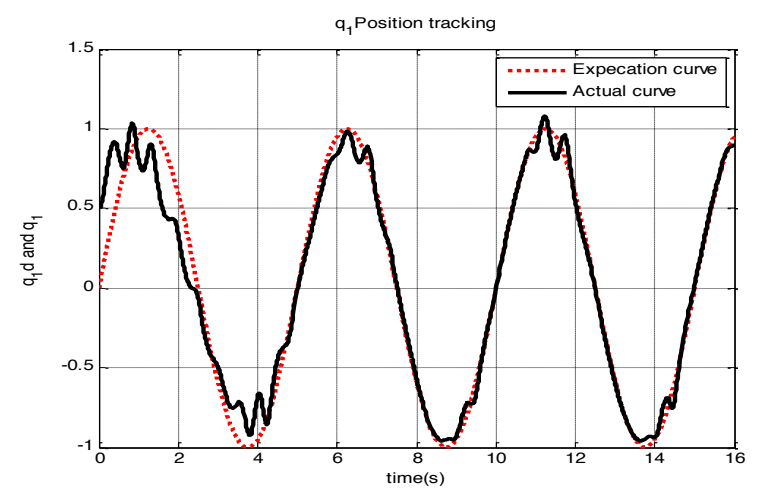

Fig. 4. Position tracking curve of $q_{1}$ at external disturbance condition

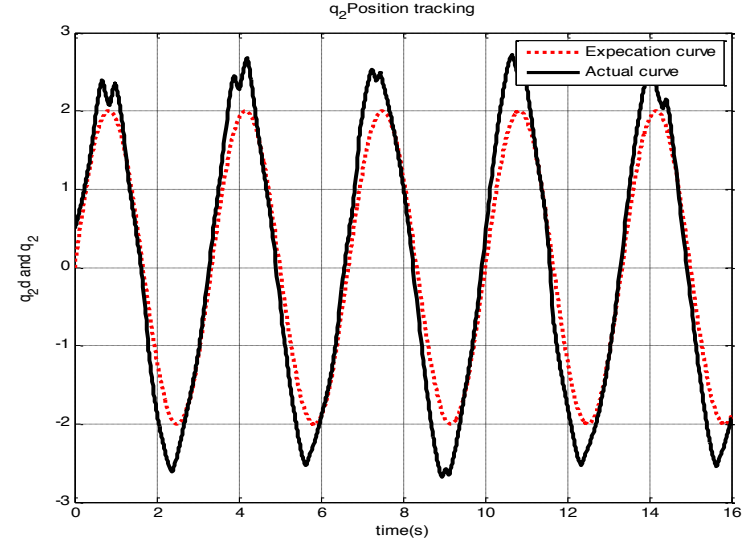

Fig. 5. Position tracking curve of $q_{2}$ at external disturbance condition

Numerical simulations demonstrate the effectiveness of the proposed fuzzy backstepping sliding control method, and there are the advantages of strong robustness and antidisturbance capacity. At external disturbance condition, the system still has good control capacity and strong robustness.

\section{Conclusions}

In this paper, by combining the Backstepping method and the T-S fuzzy model method, the fuzzy backstepping sliding mode control was presented for a class of mismatched uncertain system. In this new method, nonlinear system is described based on T-S fuzzy dynamical model, and the nonlinear system is translated into local linear model by fuzzy method, not only make the system is stable, and avoid the chattering phenomenon of sliding mode control, Then the global stability of the control law was also achieved by a Lyapunov function. Simulation results indicate that the proposed controller is valid and effective, and it has strong robustness to external disturbance and the performance of system.

\section{References}

1. Hu Y.A., Jin Y.-Q, Li H.-Y, "Robustness of adaptive backstepping control for nonlinear systems", Publishing House of Electronics Industry, Beijing, 2010, pp.1-18.

2. Bouadi H., Bouchoucha M. and Tadjine M., "Sliding Mode Control based on Backstepping Approach for an UAV Type-Quadrotor", International Journal of Mechanical, Industrial Science and Engineering 1(2), 2007, pp.11524-1-6.

3. Wang Z., "Synchronization of an Uncertain Fractional-Order Chaotic System via Backstepping Sliding Mode Control”, Discrete Dynamics in Nature and Society, 2013, pp.732503-1-6.
4. Farzin Piltan, Sulaiman N.,Iraj Asadi Talooki and Ferdosali P., "Control of IC Engine: Design a Novel MIMO Fuzzy Backstepping Adaptive Based Fuzzy Estimator Variable Structure Control”, International Journal of Robotics and Automation 2(5), 2011, pp.360-380.

5. Mohammad Reza Soltanpour, Behrouz Zolfaghari, "Fuzzy Sliding Mode Control Design for a Class of Nonlinear Systems With Structured and Unstructured Uncertainties", International Journal of Innovative Computing, Information and Control 9(7), 2013, pp.2713-2726. 
6. Abdel Badie Sharkawy, Shaaban Ali Salman, “An Adaptive Fuzzy Sliding Mode Control Scheme for Robotic Systems", Intelligent Control and Automation 2(4), 2011, pp.299-309.

7. Wang Y.-F., Jiang C.-S. "Direct adaptive fuzzy sliding mode control with variable universe for near space vehicle Systems", Engineering and Electronics 33(3), 2011, pp.633-637.

8. Farzin Piltan, N. Sulaiman, I. Asadi Talooki and P. Ferdosali, "Control of IC Engine: Design a Novel MIMO Fuzzy Backstepping Adaptive Based Fuzzy Estimator Variable Structure Control", International Journal of Robotics and Automation 2(5), 2011, pp.360-380

9. Tong S.-C., Wang T., Wang Y.-P. and Tang J.-T., "Design and stability analysis of fuzzy control system", Science Press, Beijing 2004, pp.21-50.

10. Ying H., "General SISO Takagi-Sugeno fuzzy system with linear rule consequent Are Universal approximators", IEEE Transactions on Fuzzy Systems 6(4), 1998, pp.582-587.
11. Sun C.-H., Lin S.-W. and Wang Y.-T., "Relaxed Stabilization conditions for switching T-S Fuzzy system with practical constraints", International Journal of Innovative Computing, Information and Control 8(6),2012, pp.4133-4145.

12. Mozelli L.A., Palhares R.M., Souza F.O. and Mendes E.M.A.M., "Reducing conservativeness in recent stability conditions of T-S fuzzy systems", Automatica 45(6), 2009, pp. 1580-1583.

13. Chungshi Tseng. "Fuzzy Tracking Control Design for Nonlinear Dynamic Systems via T-S Fuzzy Model”, IEEE Transactions on Fuzzy Systems 9(3), 2001, pp.381-392.

14. E. Nechadi and M.N. Harmas, "Power System Stabilizer Based on Global Fuzzy Sliding Mode Control”, Balkan Journal of electrical and computer engineering 1(2), 2013, pp.78-84

15. Lian K.-Y., Chiang T.-S., Chiu C.-S. , "Synthesis of Fuzzy ModelBased Designs to Synchronization and Secure communications for Chaotic Systems", IEEE Transactions on systems, man and Cybernetics-part B 31(1), 2001, pp.66-83. 